\title{
New Rapid Enzyme-Linked Immunosorbent Assay to Detect Antibodies against Bacterial Surface Antigens Using Filtration Plates
}

\author{
Saori Itoh, ${ }^{a}$ Masako Kariya, ${ }^{a}$ Keiji Nagano, ${ }^{a}$ Shin-ichiro Yokoyama, ${ }^{a}$ Toshio Fukao, ${ }^{b}$ \\ Yoshihisa YAMAZAKI, ${ }^{c}$ and Hiroshi MORI ${ }^{*, a}$ \\ ${ }^{a}$ Laboratory of Microbiology, Department of Public Health Pharmacy, Gifu Pharmaceutical University; 5-6-1 Mitahora- \\ higashi, Gifu 502-8585, Japan: ${ }^{b}$ Gifu Municipal Institute of Public Health; 5-2 Aoyagicho, Gifu 500-8881, Japan: and \\ ${ }^{c}$ Department of Pediatrics, Prefectural Gifu Hospital; 4-6-1 Noishiki, Gifu 500-8717, Japan. \\ Received February 21, 2002; accepted May 23, 2002
}

\begin{abstract}
An easy and rapid ELISA system, Filtration ELISA, to detect antibodies against bacterial cell surface antigens was developed using a 96-well filtration plate fitted with a $0.22 \mu \mathrm{m}$ membrane (MultiScreen ${ }^{\circledR}$-GV, Millipore). Bacterial whole cells were used as antigens without fixing the cells with formalin etc. The whole cell antigens were washed by vacuum filtration through a filter and resuspended in washing buffer. Assay reactions could be done in the wells without losing the solution. The technique was established using antisera of mice immunized with Escherichia coli, and then evaluated by assaying antibodies to Shiga-toxin producing E. coli $0157: H 7$ (STEC), Staphylococcus aureus and Lactobacillus acidophilus in fecal extracts of 157 children who had eaten school lunches contaminated with STEC in comparison with 25 age-matched control children. The lunch group showed significantly higher IgA antibody titers against STEC than the control group $(p<0.0005)$, but not against L. acidphilus. The results indicate that Filtration ELISA is a quantitative and specific technique for measuring antibodies against antigens on the surface of bacteria without extracting antigens from the bacteria. This technique is widely applicable to the assay of antibodies in various samples including serum and fecal extract against various kinds of bacteria.
\end{abstract}

Key words ELISA; bacterial surface antigen; antibody; Shiga-toxin producing Escherichia coli; O157; feces

Antibodies against bacteria are assayed for many purposes such as diagnosing bacterial infections, epidemiological studies, and developing new vaccines. ${ }^{1-3)}$

Various methods are used to assay antibodies against bacteria including: immuno-precipitation, ${ }^{4)}$ immuno-agglutination, immunoblotting, ${ }^{5,6)}$ radioimmunoassay, ${ }^{7,8}$ and enzyme linked immunosorbent assay (ELISA). ELISA is used most frequently because it is easy, rapid and has high sensitivity. Antigens for ELISA detection of antibodies usually include plastic-fixable substances such as soluble proteins and DNA, even antigens fixed to plastic by antibodies against them. Therefore, fixable antigens have been extracted from bacteria by methods of sonication, treatment with detergents and so on. Extraction processes are always accompanied with the destruction of the cell, ${ }^{5,9-11)}$ and extracts contain not only cell surface antigens but also such cellular ingredients as cytoplasmic proteins. These kinds of crude extracts are often used without purification to titrate antibodies. However, unpurified extracts are not always appropriate as antigens for the ELISA detection of immuno-protective antibodies against bacterial infection, while antibodies against bacterial cell surface antigens are suitable, those against cytoplasmic components are not. In addition, impurities may competitively inhibit fixation of subjective cell surface antigens to plastic surfaces.

Bacteria have many antigens on their cell surfaces. Bacterial surface antigens such as outer membrane proteins, ${ }^{12-15)}$ flagella $^{14)}$ and lipopolysaccharides (LPS) ${ }^{9,12-14)}$ are often purified to detect anti-bacterial cell surface antibodies. However, it can be unclear which antibody should be assayed for the detection of surface antigens that are related to immunity. To better clarify this difficulty and reduce the labor involved in purification, whole bacterial cells were used as antigens to detect anti-bacterial cell surface antibodies by directly fixing them physically on plates. ${ }^{16-18)}$

In the present study, we developed an easy and rapid ELISA system to detect antibodies against bacterial cell surfaces with neither isolating the antigens from bacteria nor fixing bacteria on plates by physical methods. The method employed 96-well filtration plates with a $0.22 \mu \mathrm{m}$ membrane (MultiScreen ${ }^{\circledR}-G V$, Millipore) to maintain bacterial cells in the wells as antigens.

To confirm the efficacy of the method developed, Filtration ELISA was applied to fecal samples collected from 157 children who had eaten school lunches contaminated with Shiga toxin-producing Escherichia coli (STEC, serotype O157:H7) during an outbreak of food poisoning in Gifu, Japan, in 1996. The results indicate that Filtration ELISA is a quantitative and specific technique for measuring antibodies against various types of bacteria, which is quick and easy to use for large numbers of samples without extracting antigens.

\section{MATERIALS AND METHODS}

Bacterial Strains and Growth Conditions Shiga toxin 1 and 2 producing E. coli GPU96MM (serotype O157:H7) was isolated from stool samples of a patient with hemorrhagic colitis during an outbreak of disease in Gifu, Japan, in June 1996. The other bacterial strains used in this study were E. coli GY4015 (K12) obtained from C.N.R.S., Gif-surYvette, France, E. coli IID5208 (O1:H7, non-pathogenic) and Staphylococcus aureus IID980 purchased from Institute of Medical Science, Tokyo University, Tokyo, Japan, and Lactobacillus acidophilus GAI obtained from Institute of Anaerobic Bacteriology, School of Medicine, Gifu University, Japan. L. acidophilus GAI were grown in BBL LBS broth (BectonDickinson Microbiology Systems, Sparks, MD, U.S.A.), and the other strains of bacteria were cultured in nu- 
trient broth (Nissui Pharmaceutical Co., Tokyo) consisting of $5 \mathrm{~g} / 1$ meat extract, $15 \mathrm{~g} / 1$ peptone, $5 \mathrm{~g} / 1 \mathrm{NaCl}$ and $5 \mathrm{~g} / 1 \mathrm{KH}_{2} \mathrm{PO}_{4}$. Cells in logarithmic growth were harvested by centrifugation at $4500 \times \boldsymbol{g}$ for $15 \mathrm{~min}$ and washed three times with sterile phosphate buffered saline (PBS, $\mathrm{pH} 7.4$ ) containing $0.1 \%$ sodium azide $\left(\mathrm{NaN}_{3}-\mathrm{PBS}\right)$. These cells were used as antigens for immunizing mice and/or for Filtration ELISA.

Filtration ELISA Washing buffer used in the assay was PBS containing $0.1 \%$ Tween 20 . Blocking buffer-A consisted of PBS containing 1\% bovine serum albumin (BSA, Fraction $\mathrm{V}$, Nacalai Tesque Inc.), 5\% normal calf serum (NCS, Irvin Scientific, Kogan, UT, U.S.A.), $0.1 \%$ Tween 20 and $0.1 \%$ $\mathrm{NaN}_{3}$. Blocking buffer-B consisted of PBS containing 1\% BSA, 5\% NCS, $0.1 \%$ Tween 20 and $0.01 \%$ thimerosal. All buffers were filtrated through a Sterivex-GS filter $(0.22 \mu \mathrm{m}$, Millipore, Bedford, MA, U.S.A.) before use.

Bacterial cells suspended in $\mathrm{NaN}_{3}$-PBS were counted using a Petroff-Hausser hemocytometer for bacteria (Sunlead Glass Co., Tokyo) with gentiana violet stain, and diluted with $\mathrm{NaN}_{3}$-PBS to an appropriate density. The cell suspension in a volume of $200 \mu \mathrm{l}$ was applied over the filter of the filtration plate wells (MultiScreen ${ }^{\circledR}-\mathrm{GV}, 96$-well membrane plate, pore size $0.22 \mu \mathrm{m}$, Millipore). After being subjected to vacuum filtration with a commercially available instrument (MAVM09601, Millipore), the cells were incubated with $200 \mu \mathrm{l}$ of blocking buffer-A supplemented with $0.4 \mu \mathrm{l} / \mathrm{ml}$ of $30 \% \mathrm{H}_{2} \mathrm{O}_{2}$ at $37^{\circ} \mathrm{C}$ for $1 \mathrm{~h}$ to inactivate endogenous cellular peroxidases. Then, the buffer was removed and $200 \mu \mathrm{l}$ of washing buffer was added to the wells and cells were resuspended with a microplate shaker. For this assay, whole cell antigens were washed by vacuum filtration through a filter and resuspended in washing buffer. After inactivating endogenous peroxidases, the cells were incubated with test samples (mouse antiserum or fecal sample extracts) diluted in blocking buffer-A for $2 \mathrm{~h}$ at $37^{\circ} \mathrm{C}$ in a volume of $100 \mu \mathrm{l}$. Then, the cells were washed 4 times, and incubated with peroxidase-labeled anti-mouse or human immunoglobulin antibodies diluted with blocking buffer-B. After washing 5 times, the wells were subjected to enzymatic reaction for $30 \mathrm{~min}$ at $37^{\circ} \mathrm{C}$ with $150 \mu \mathrm{l}$ substrate solution ( $\mathrm{pH} 5.0$ ) consisting of $0.05 \mathrm{M}$ citric acid, $0.1 \mathrm{M}$ disodium hydrogen phosphate, 0.4 $\mathrm{mg} / \mathrm{ml}$ of $o$-phenylene diamine and $0.4 \mu \mathrm{l} / \mathrm{ml}$ of $30 \% \mathrm{H}_{2} \mathrm{O}_{2}$. The reaction was terminated by adding $50 \mu \mathrm{l}$ of $2 \mathrm{M} \mathrm{H}_{2} \mathrm{SO}_{4}$. Aliquots $(100 \mu \mathrm{l})$ of the resulting solution were transferred to transparent 96-well flat-bottom microplates. OD at $492 \mathrm{~nm}$ (reference $660 \mathrm{~nm}$ ) was measured for the transferred solution with a microplate-reader (MTP-120, CORONA ELECTRIC Co., Ibaragi, Japan). Wells exhibiting OD values over 3.0 were all considered to be the maximum value of 3 . To examine background OD values, blocking buffer-A was used in place of test samples. All assays were carried out in duplicate, and the average of duplicate OD measurements at the defined dilution of test samples was considered the antibody titer.

Antibodies used as ELISA reagents were as follows. Peroxidase-conjugated goat anti-mouse IgM (mu-chain specific) (PO-GaM IgM), and peroxidase-conjugated goat anti-mouse IgA (alpha-chain specific) (PO-GaM IgA) were purchased from ZYMED Laboratories, San Francisco, CA, U.S.A. Peroxidase-conjugated goat affinity purified antibody to mouse IgG Fc (PO-GaM IgG) was purchased from ORGANON
TEKNIKA Corporation, West Chester, PA, U.S.A.

Peroxidase-conjugated $\mathrm{F}\left(\mathrm{ab}^{\prime}\right)_{2}$ fragment of rabbit antihuman IgA (specific for alpha-chain) (PO-RaH IgA) was purchased from DAKO Corporation, Carpinteria, CA. By conventional ELISA using commercially available purified mouse or human IgM, IgG and IgA as antigens, the antibodies used in this study were confirmed to have satisfactory immunoglobulin class specificity.

Preparation of Mouse Antisera to $E$. coli GPU96MM and $\boldsymbol{E}$. coli GY4015 E. coli GPU96MM and/or $E$. coli GY4015 cells were killed by maintaining them in PBS containing $0.2 \%$ formalin for $3 \mathrm{~d}$ at $4{ }^{\circ} \mathrm{C}$. Dead, washed cell suspensions were emulsified with an equal amount of Freund's incomplete adjuvant (Nacalai Tesque Inc., Kyoto, Japan). ICR female mice (Japan SLC, Inc., Hamamatsu, Japan), 6 weeks old, were immunized with intraperitoneal and subcutaneous injections totaling $0.2 \mathrm{ml}$ of the emulsion $\left(10^{7}\right.$ cells $)$, and boosted $7 \mathrm{~d}$ later in the same manner. Antisera obtained from 8 immunized mice 1 week after the final immunization were pooled, and $20 \mu \mathrm{l}$ aliquots of each were stored at $-80^{\circ} \mathrm{C}$ until use.

Human Fecal Samples and Extraction Fecal samples were obtained from 157 children (6 to 12 years of age) that had eaten school lunches contaminated with $E$. coli GPU96MM on June 5, 1996 at Nagamori elementary school in Gifu. Among the subjects, 41 children suffered from abdominal pain without diarrhea, and 35 had diarrhea with abdominal pain. The other 81 children showed no symptoms. The majority of samples were collected from 7 to $13 \mathrm{~d}$ after eating the contaminated lunches. Fecal samples were transported 3 times a day from the school to our institute using a cooling container, frozen at $-20^{\circ} \mathrm{C}$ within $4 \mathrm{~h}$ at longest after evacuation, and then stored at $-80^{\circ} \mathrm{C}$. Fecal samples from 25 children ( 6 to 12 years of age) who resided far from the school and who had not taken the contaminated lunches were used as controls. Control fecal samples were cooled on ice just after evacuation, and frozen within $1 \mathrm{~h}$ at $-80^{\circ} \mathrm{C}$ until use.

To extract immunoglobulins, the fecal samples were weighed and suspended using a glass rod in a 10 fold volume of $\mathrm{NaN}_{3}$-PBS. Supernatants were separated by centrifugation of the suspension at $20000 \times \mathbf{g}$, at $4{ }^{\circ} \mathrm{C}$ for $15 \mathrm{~min}$ to be used as fecal sample extracts, and subjected to antibody assays.

Pre-absorption of Fecal Sample Extracts with Bacteria A $100 \mu \mathrm{l}$ suspension of $2 \times 10^{8} / \mathrm{ml} \mathrm{E}$. coli IID5208 and $/$ or $S$. aureus IID980 was placed over the filter of the filtration plate well. After vacuum filtration of the suspension into the wells, remaining cells were suspended with $150 \mu$ l of a 5 fold dilution of fecal sample extracts/supernatants with blocking buffer-A, followed by incubation for $2 \mathrm{~h}$ at $37^{\circ} \mathrm{C}$. The solution in the well was passed through the membrane by vacuum and recovered into another plate settled under the filtration plate. The absorbed solution was diluted 2 fold with blocking buffer-A to be assayed by Filtration ELISA at a final 10 fold dilution.

Statistical Analysis Significance of differences between the control and the contaminated lunch groups was analyzed by Student's or Aspin-Welch's two-tailed $t$-test after $F$-test to examine homogeneity between the two groups. Significance of correlation coefficient (r) was analyzed by calculating $t$ value. A value of $p \leq 0.05$ was considered to be statistically 
significant in all statistical analyses.

\section{RESULTS}

Filtration ELISA Applied to Mice Antisera Anti-E. coli GY4015 mouse antiserum was subjected to Filtration ELISA to detect IgG antibody against $E$. coli GY4015 cells at various cell densities (Fig. 1). The OD value increased together with the number of cells as antigens, and reached to almost a maximun at $1 \times 10^{7}$ cells/well. Background values increased at $4 \times 10^{7}$ cells/well, but they remained within negligible levels with any of the cell numbers examined.

IgM, IgG and IgA antibodies were titrated for serial dilutions of anti-E. coli GY4015 mouse antiserum (Fig. 2). The $\mathrm{OD}$ values for IgM and IgG antibodies increased in a concentration dependent manner. OD values for IgA antibodies did not increase even at the highest antiserum concentration.

To examine the specificity of antibody detection by Filtration ELISA, anti-E. coli GY4015 and anti-E. coli GPU96MM mice antisera were subjected to the assay against $E$. coli GY4015 and E. coli GPU96MM cells in the manner of crossing the antigens and antisera (Fig. 3). The OD values for IgM and $\mathrm{IgG}$ antibodies in anti-E. coli GY4015 antiserum increased against E. coli GY4015 but not against E. coli GPU96MM. On the other hand, the OD value for IgM antibody in anti-E. coli GPU96MM antiserum increased against E. coli GPU96MM but not against E. coli GY4015. IgG antibody in anti-E. coli GPU96MM antiserum was not detected against $E$. coli GPU96MM.

Filtration ELISA Applied to Human Fecal Samples Filtration ELISA was applied to the fecal samples collected from 157 children who had eaten lunches contaminated with E. coli GPU96MM, and from 25 control children. Fecal IgA antibody titers were assayed at a 10 fold final dilution of the sample extracts against E. coli GPU96MM, S. aureus IID980, and L. acidophilus GAI (Fig. 4A). Statistical differences were observed between the control and contaminated lunch groups in the antibody titer against $E$. coli GPU96MM at $p<0.0005$, and that against $S$. aureus IID980 at $p<0.02$. There was no statistical difference in the antibody titer against $L$. acidophilus GAI between the two groups.

The filtration plate used here allows for the recovery of filtered solution from each of the 96 wells. Using this plate, fecal sample extracts were pre-absorbed with $E$. coli IID5208 and/or S. aureus IID980 to remove antibodies against common bacterial antigens. The absorbed solutions were then subjected to Filtration ELISA to evaluate IgA antibody titer against $E$. coli GPU96MM at a 10 fold final dilution of the fecal sample extracts (Fig. 4B) as well as the non-absorbed extracts (Fig. 4A).

The IgA antibody titers against E. coli GPU96MM in fecal sample extracts of the control group were markedly lower following absorption with $E$. coli IID5208 and/or $S$. aureus IID980 in contrast to those from the contaminated lunch group. Also the statistical differences between the two groups were greater at $p<0.0001$ in both cases of pre-absorption with $E$. coli IID5208 and $S$. aureus IID980. The antibody titers after absorption with E. coli IID5208 and $S$. aureus IID980 showed good linear correlation with the contaminated lunch group (Fig. 5).

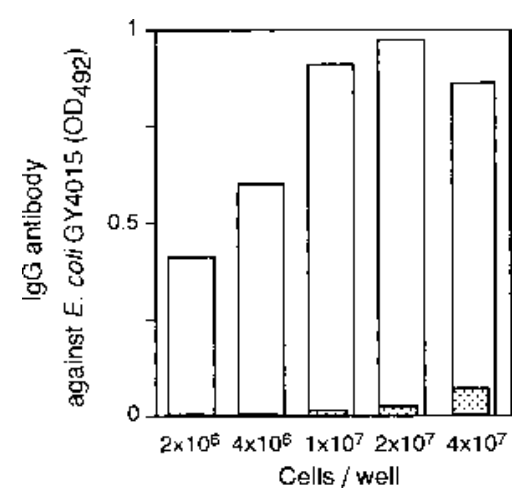

Fig. 1. Anti-E. coli GY4015 IgG Antibody in Mouse Antiserum Detected at Various Cell Densities of E. coli GY4015 in a Well

The antiserum was assayed at a dilution of $1: 8000$. Peroxidase-conjugated goat antimouse IgG antibody (PO-GaM IgG) was used at a dilution of $1: 500$. Columns represent the means of duplicate sets. Dotted columns represent background where the dilution buffer was used instead of the antiserum.

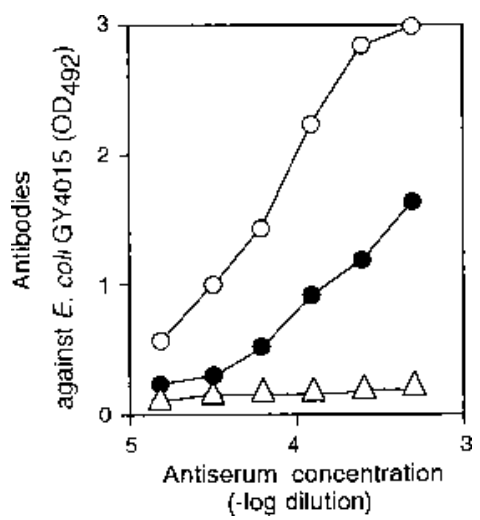

Fig. 2. Titration Curves for IgM, IgG and IgA Antibodies in Anti-E. coli GY4015 Mouse Antiserum at Serial Dilutions from 1:2000 to $1: 64000$

E. coli $\mathrm{GY} 4015$ was used as antigen at $2 \times 10^{7}$ cells/well. Peroxidase-conjugated goat anti-mouse IgM, IgG and IgA antibodies (PO-GaM IgM, PO-GaM IgG and PO-GaM IgA) were used at a dilution of $1: 500$. Points represent the means of duplicate sets. Open circle: IgM, Closed circle: IgG, Open triangle: IgA.

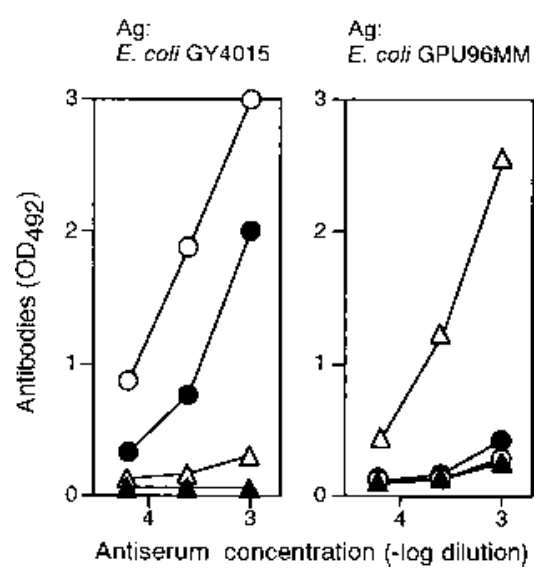

Fig. 3. IgM and IgG Antibodies Detected in Anti-E. coli GY4015 and E. coli GPU96MM Mouse Antisera at Serial Dilutions from $1: 1000$ to $1: 32000$

The cells as the antigen $(\mathrm{Ag})$ were used at $2 \times 10^{7}$ cells/well. Peroxidase-conjugated goat anti mouse IgM and IgG antibodies (PO-GaM IgM and PO-GaM IgG) were used at a dilution of $1: 500$. Points represent the means of duplicate sets. Open and closed circles: anti-E. coli GY4015 antiserum developed with PO-GaM IgM, and PO-GaM IgG, respectively. Open and closed triangles: anti-E. coli GPU96MM antiserum developed with PO-GaM IgM and PO-GaM IgG, respectively. 
A

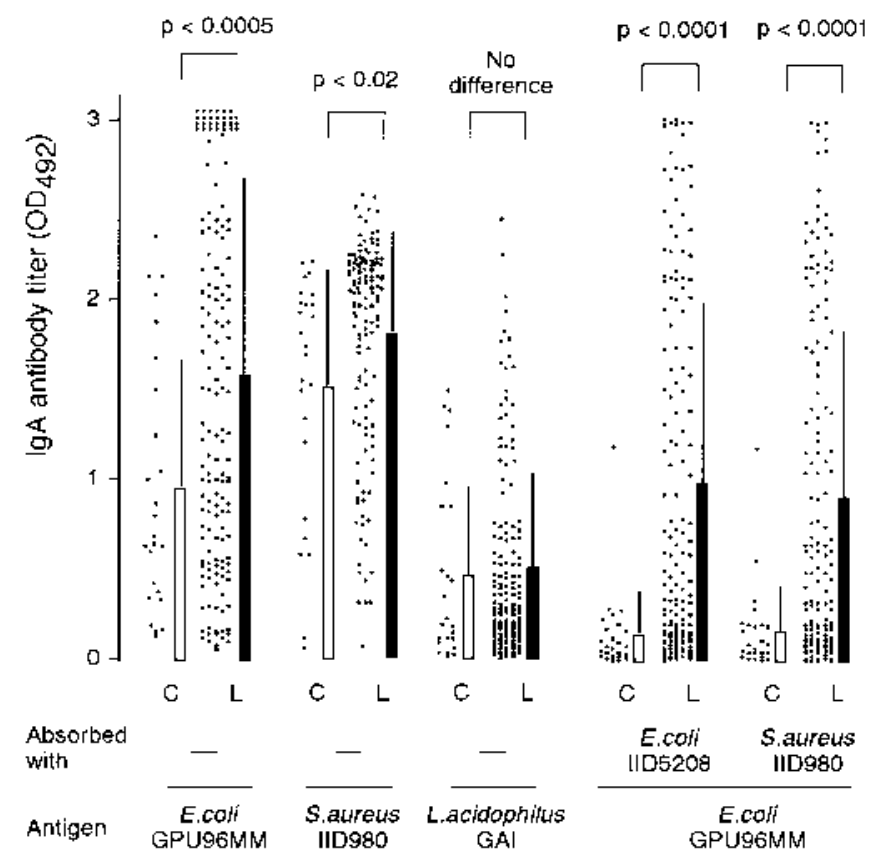

Fig. 4. IgA Antibody against E. coli GPU96MM, S. aureus IID980 and $L$. acidophilus GAI in Fecal Samples Collected from 157 Children Following an Outbreak of STEC (L) and 25 Control Children (C)

The feces of children were extracted with a 10 fold volume of PBS. Antibody titers in the extracts were assayed by Filtration ELISA at a 10 fold final dilution of the extracts. Whole bacterial cells as the antigen were used at $2 \times 10^{7}$ cells/well. Panel A: nonabsorbed. Panel B: absorbed with E. coli IID5208 or S. aureus IID980. Dots represent individual children. Columns and vertical bars represent the mean and standard deviation (S.D.).

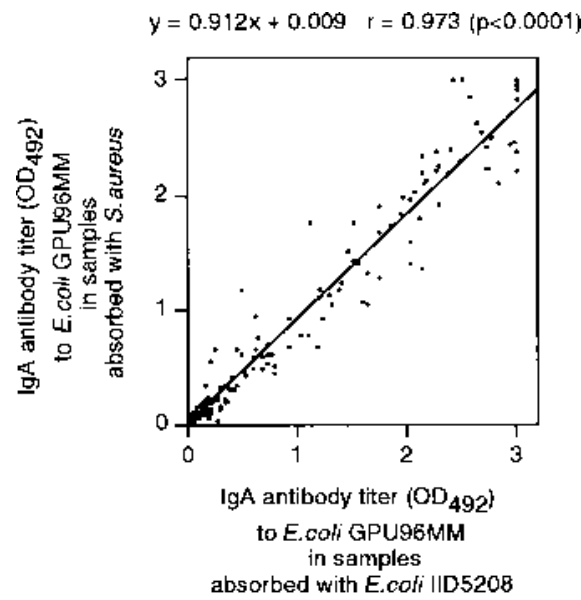

Fig. 5. Correlation between $\operatorname{IgA}$ Antibody Titers against E. coli GPU96MM after Absorption with E. coli IID5208 and with S. aureus IID980 in the Extracts of Fecal Samples Collected from 157 Children Following an Outbreak of STEC

Dots represent individual children

\section{DISCUSSION}

The bacteria were used as antigens in Filtration ELISA without killing or fixing them with formalin etc. in order to avoid denaturing the antigens on their surfaces, although they were inhibited from the proliferation with $\mathrm{NaN}_{3}$ added into the ELISA buffers. But much lower risk of environmental contamination by the bacteria is associated with Filtration
ELISA, since cells are washed by vacuum filtration, and both the cells and the solution can be manipulated in closed fashion.

Almost identical OD values were produced for IgG antibody to $E$. coli GY4015 at the same antisera dilutions as shown in Figs. 1, 2 and 3 from three independent experiments, indicating that this method provides satisfactory reproducibility for titrations. The results shown in Fig. 3 indicate that good specificity of this method for detecting antibodies. It is unknown why $\mathrm{IgG}$ antibody to $E$. coli GPU96MM was not produced in mice immunized with the bacteria (Fig. 3 right panel) in contrast to IgG antibody to $E$. coli GY4015 (Fig. 3 left panel). E. coli GPU96MM antigens, especially a main surface antigen of O157-LPS, may not cause immunoglobulin class switch in the immune response.

Earlier experiments confirmed that blocking buffer-A containing $\mathrm{NaN}_{3}$ inhibits bacterial proliferation during assay incubation (data not shown). Endogenous bacterial peroxidases were inactivated by incubating for $1 \mathrm{~h}$ at $37^{\circ} \mathrm{C}$ with blocking buffer-A supplemented with hydrogen peroxide, as shown by the negligible background level (Fig. 1). Thus, Filtration ELISA allows for the measurement of antibodies against bacterial cell surface antigens, which is both easy and rapid, for large numbers of samples without the extraction of antigens.

We applied Filtration ELISA to assay antibodies in fecal samples of children against $E$. coli GPU96MM (O157:H7), $S$. aureus IID980 and L. acidophilus GAI in order to evaluate the ELISA system. In this experiment, we focused only on IgA antibody titers against them, because it is known that antibodies present in feces as a result of intestinal secretion are predominantly polymeric IgA antibodies. Our results (Fig. 4A) disclosed that IgA antibody against E. coli GPU96MM increased markedly in the contaminated lunch group. STEC as well as enteropathogenic $E$. coli adhere to host intestinal epitherial cells through a type III secreting mechanism and cause intimate and effacement $(\mathrm{A} / \mathrm{E})$ lesion. Pathogenic factors concerning with the type III secreting mechanism and the following adherence are all encoded on locus of enterocyte effacement (LEE, $45 \mathrm{kbp}$ ). ${ }^{19-21)}$ Intimin, a protein encoded by eae gene in LEE, is expressed on the outer membrane of the bacteria ${ }^{22)}$ as a ligand for Tir that is secreted and translocated into host cells through the type III secreting apparatus. An EspA filament encoded by espA gene in LEE is also known to be expressed on the outer membrane of the bacteria, and form a needle structure through which various proteins including Tir are secreted. ${ }^{23,24)}$ Then, Filtration ELISA performed here might detect the antibodies against these proteins expressed on the surface of the bacteria such as intimin and EspA in addition to the main surface antigen of O157-LPS. It is remaining to be studied in future, whether the fecal samples contain antibodies against such pathogenic proteins concerning $\mathrm{A} / \mathrm{E}$ lesion and whether the antibodies are protective to the development of the disease.

An increase in antibody against $S$. aureus was also seen, but not against L. acidophilus in the contaminated lunch group (Fig. 4A). S. aureus are indigenous bacteria with the pathogenicity in nasal cavity. On the other hand, L. acidophilus are indigenous bacteria without the pathogenicity in intestine. It is probable that human is commonly immunized with $S$. aureus to prevent its pathogenicity. In the following 
paper, we will describe that the increase of $\operatorname{IgA}$ antibody against $S$. aureus might be a result of non-specific stimulation of the primed antibody production in acute inflammatory phase of the STEC infection.

It is well known that there are many common antigens on the cell surfaces of various bacterial strains. Therefore, we carried out absorption experiments. Absorption is one possible application of the present method, since antibodies which are not fixed to bacteria can be recovered from reaction mixtures in individual wells. In both cases of absorption with $E$. coli IID5208 and $S$. aureus IID980, antibody titers against $E$. coli GPU96MM in the control group decreased substantially, and the difference in antibody titers between the control and contaminated lunch groups became more pronounced (Fig. 4B). The IgA antibody titers after absorption with $E$. coli IID5208, a gram-negative enterobacterium, showed a strict correlation with those after absorption with $S$. aureus IID980, a gram-positive coccus (Fig. 5). This result of the good correlation suggests that common bacterial antigens are prevalent among a wide spectrum of bacteria, and the absorption procedure increases the specificity to detect antibodies against the target bacteria.

Thus, Filtration ELISA provides a quantitative and specific technique for measuring antibodies against antigens on the surface of bacteria without extracting antigens from the bacteria. This technique is widely applicable to the assay of antibodies in various samples including serum and fecal extract against various kinds of bacteria.

\section{REFERENCES}

1) Jertborn M., Svennerholm A. M., Holmgren J., Vaccine, 14, 14591465 (1996).

2) Bhattacharjee A. K., Opal S. M., Taylor R., Naso R., Semenuk M., Zollinger W. D., Moran E. E., Young L., Hammack C., Sadoff J. C., Cross A. S., J. Infect. Dis., 173, 1157-1163 (1996).

3) Jertborn M., Svennerholm A.-M., Holmgren J., Vaccine, 12, 1078 1082 (1994).

4) Elkins C., Barkley K. B., Carbonetti N. H., Coimbre A. J., Sparling P.
F., Mol. Microbiol., 14, 1059-1075 (1994).

5) Wittenbrink M. M., Failing K., Krauss H., Vet. Microbiol., 48, 257268 (1996).

6) Wilske B., Fingerle V., Preac-Mursic V., Jauris-Heipke S., Hofmann A., Loy H., Pfister H.-W., Rossler D., Soutschek E., Med. Microbiol. Immunol. Berl., 183, 43-59 (1994).

7) Borrelli S., Roggen E. L., Hendriksen D., Jonasson J., Ahmed H. J., Piot P., Jansson P. E., Lindberg A. A., Infect. Immun., 63, 2665-2673 (1995).

8) Mitchell P. D., Reed K. D., Aspeslet T. L., Vandermause M. F., Melski J. W., J. Clin. Microbiol., 32, 1958-1962 (1994).

9) Ou J. G., Bainbridge B., Gu K., Sims T. J., Whitney C. W., Darveau R. P., Chen H. A., Houston L. S., Page R. C., Oral Microbiol. Immunol., 12, $11-19$ (1997).

10) Plum G., Brenden M., Santos P., Schwarz E., Wahnschaffe U., Mauff G., Pulverer G., Zentralbl. Bakteriol., 284, 348-360 (1996).

11) Prescott J. F., Fernandez A. S., Nicholson V. M., Patterson M. C., Yager J. A., Viel L., Perkins G., Equine Vet. J., 28, 344-349 (1996).

12) Rahman M., Holme T., Jonsson I., Krook A., APMIS, 105, 213-220 (1997).

13) Rodriguez Barbosa J. I., Gutierrez Martin C. B., Tascon R. I., Gonzalez O. R., Mittal K. R., Rodriguez Ferri E. F., FEMS Immunol. Med. Microbiol., 16, 173-181 (1996).

14) Bangsborg J. M., Shand G. H., Hansen K., Wright J. B., APMIS, 102, 501-508 (1994).

15) Gerber M. A., Shapiro E. D., Bell G. L., Sampieri A., Padula S. J., J. Infect. Dis., 171, 724-727 (1995).

16) Torres J. F., Lyerly D. M., Hill J. E., Monath T. P., Infect. Immun., 63, 4619-4627 (1995).

17) Chen D., McMichael J. C., VanDerMeid K. R., Hahn D., Mininni T., Cowell J., Eldridge J., Infect. Immun., 64, 1900-1905 (1996).

18) Siddons C. A., Chapman P. A., J. Med. Microbiol., 39, 408-415 (1993).

19) Perna N. T., Mayhew G. F., Posfai G., Elliott S., Donnenberg M. S., Kaper J. B., Blattiner F. R., Infect. Immun., 66, 3810-3817 (1998).

20) Groisman E. A., Ochman H., Cell, 87, 791—794 (1996).

21) McDaniel T. K., Jarvis K. G., Donnenberg M. S., Kaper J. B., Proc. Natl. Acad. Sci. U.S.A., 92, 1664-1668 (1995).

22) Louie M., Azavedo J. C. S., Handelsman M. Y. C., Clark C. G., Ally B., Dytoc M., Sherman P., Brunton J., Infect. Immun., 61, 4085-4092 (1993).

23) Knutton S., Rosenshine I., Pallen M. J., Nisan I., Neves B. C., Bain C., Wolff C., Dougan G., Frankel G., EMBO J., 17, 2166-2176 (1998).

24) Ebel F., Podzadel T., Rohde M., Kresse A. U., Kramer S., Deibel C., Guzman C. A., Chakraborty T., Mol. Microbiol., 30, 147-161 (1998). 